\title{
CO-VID-EO: resilient hybrid learning strategies to explicitly teach team skills in undergraduate students.
}

\author{
Qingqi Wang ${ }^{1}$ and Amanda Rasmussen ${ }^{1}$ \\ ${ }^{1}$ University of Nottingham
}

July 20, 2020

\begin{abstract}
The modern workplace requires teamwork bridging distances, time-zones and cultures using virtual collaboration platforms. As such teamwork is a key employability skill for our graduates, and yet we rarely explicitly teach team skills or how to navigate and collaborate in a virtual environment. Here we demonstrate that integrating in-person (synchronous) and online (synchronous and asynchronous) learning environments can prove an extremely resilient teaching method that allowed continuity during COVID-19 lockdown, while providing them with these essential skills. Students were given a semester long documentary video team assignment supported by regular compulsory team training sessions and using the Microsoft Teams online collaboration platform. At the end of semester, the 24 students in the class were sent a survey with questions relating to their perspectives of teamwork in general, the team training sessions and online platform and the impact of COVID-19. Of the 12 respondents only 4 reported negative attitudes to teamwork in general and learning and shared workload were the most reported benefits. Implementing explicit (and compulsory) team training sessions throughout semester were valued by the students for reasons ranging from the explicit intention of each task (team establishment and planning, negotiation, reflection of team behaviours, negotiating team member contributions) to the more general appreciation that regular compulsory sessions ensured the teams met frequently. It was particularly positive that every student reported that this team experience was better than their previous team experiences, and this in spite of the COVID-19 lockdown. Included with this manuscript are all the learning materials provided to the students and a few key lessons we learnt along the way.
\end{abstract}

\section{INTRODUCTION}

Teamwork is an essential employability skill expected of our graduates across a range of disciplines (García et al., 2016; Wilson et al., 2018). In addition to the requirement of team skills for the workplace, much research has demonstrated that students can learn more when working in effective teams (Wilson et al., 2018). Critical thinking, engagement, cohesion, respect for diversity, mental health and interpersonal skills have all been shown to improve via team learning experiences (Chu et al., 2019; García et al., 2016; Gillespie, 2012; Gleadow et al., 2015; Mercer-Mapstone \& Kuchel, 2015; Wilson et al., 2018). The highest learning gains can be obtained when groups contain diversity, students are held accountable, assignments are designed to encourage working together, and teams receive frequent feedback (Allan, 2016; Gillespie, 2012; Rasmussen et al., 2011). While students also cite the benefit of learning team skills they often feel insufficiently trained in these skills by their degree (Wilson et al., 2018). It is therefore important for educators to consider appropriate support to facilitate the learning of these essential employability skills (García et al., 2016; Kuchel et al., 2014; Wilson et al., 2018).

The face of teamwork is also changing with technological advances. Not only do our graduates need to learn to work within teams bridging spatially separated locations and time-zones (Ayoko et al., 2012; Jimenez et al., 2017; Panteli et al., 2019), they also need to be adaptable to new technological interfaces. Recently collaboration spaces like Microsoft Teams, Google drive and Slack have integrated file sharing, co-editing, text chats and video/audio call options enabling the work environment to be almost entirely online (Jimenez 
et al., 2017). These virtual collaboration spaces are also widely applied within research environments. The authors regularly use Microsoft Teams (mostly for within-institution collaborations and teaching) and Google drive and Slack for inter-institution research collaborations bridging international boundaries. Despite this widespread application and importance including for the research environment, we rarely teach our undergraduate students to effectively implement these technologies (García et al., 2016).

Virtual work environments are incredibly flexible and resilient to changed circumstances (Jimenez et al., 2017; Panteli et al., 2019). For example they allow users to work synchronously on a document (such as via Microsoft 365 or Google drive programs) or through video calls and screen sharing regardless of where the users are located (Panteli et al., 2019). Similarly, they allow for easy asynchronous collaboration with updates from other users easily visible and host folder sharing within the platform. This flexibility in work 'location' using online collaboration spaces allows for more flexible work arrangements to accommodate those with caring responsibilities or health challenges (Jimenez et al., 2017; Panteli et al., 2019).

While allowing flexibility, virtual teams have been associated with reduced cohesiveness caused by differences in culture, language, and technological fluency (Panteli et al., 2019). The result of an entirely online work environment is that team members may never get to know each other which can create trust and anxiety issues (Panteli et al., 2019). These challenges emphasise the need to provide training opportunities within these environments for both interpersonal and technological skills development in our students to maximise their potential for future success in these virtual team environments.

The flexibility features mentioned above also make online collaboration spaces an effective way for students to interact with learning material when not in class. Students have increasing pressures on their time from part time work to caring duties and are increasingly choosing online lectures where possible for the increased flexibility that it affords (Gleadow et al., 2015). Learning environments with both face-to-face (synchronous) and online (synchronous or asynchronous) opportunities has been demonstrated to improve student engagement (Chen \& Chiou, 2014; McFarlin, 2008; Singh, 2017). By incorporating both synchronous and asynchronous features students are able to access the material (including voice and video captures) at times that suit them and can ask questions using online forums. At Nottingham we have been using Moodle (virtual learning environment) and Echo360 lecture capture for asynchronous online learning. Lecture slides, reading materials and task instructions can be uploaded and the forum feature allows for students to ask questions where everyone can see the instructor's response. More recently we have access to Microsoft Teams (Microsoft 365 software) collaboration space and here we show how this software can be used to provide a flexible, resilient learning environment, while also teaching the students important employability skills.

As mentioned above, students often feel insufficiently trained in team skills and often dislike teamwork. Problems tend to include arranging meetings outside class, unequal contributions "social loafing" and unfair grading (Rasmussen et al., 2011; Wilson et al., 2018). However appropriate scaffolding to support students and ensure accountability can improve the student perspectives of teamwork (Rasmussen et al., 2011). We previously developed a set of team training sessions to explicitly teach students what's expected of them when working with others. These are based on, and programmed around the four stages of team function forming, storming, norming and performing (Ayoko et al., 2012). This team training package was originally designed for first year undergraduate biology students (BIOl1030) at the University of Queensland (Kuchel et al., 2014; Rasmussen et al., 2011). Their team assignment was also semester-long with the end point a 5 minute documentary video on environmental issues in South East Queensland (Kuchel et al., 2014; Rasmussen et al., 2011). Within this context the team training sessions benefited the students with at least one third of the 600 students finding each session useful (Rasmussen et al., 2011).

The success of these four team training sessions demonstrates the value of providing structured support for these transferable, non-science skills. With this in mind, we adapted these sessions to fit a new semester-long team assessment (also a documentary video) within a $3^{\text {rd }}$ year undergraduate course at the University of Nottingham, UK. Additionally the students were provided a training session on using Microsoft Teams for collaboration and sections were created within their Class Notebook (within Microsoft Teams) to guide them through the different tasks required to complete the assessment (including templates for team meetings, and 
spaces with other resources such as GDPR (General Data Protection Regulation) rules and risk assessment forms). This multifaceted scaffolding was to enable students to access the information when it was most needed - both synchronously (either in class with the lecturer or their team-mates) and asynchronously.

Unexpectedly the spring 2020 semester suffered a major interruption to in-person teaching as a result of lockdown to slow the spread of COVID-19. This meant the flexibility of the students, teacher and software was tested to extremes. Here we investigate the student perspective of teamwork within the context of a hybrid course (module) integrating synchronous and asynchronous, in-person and online collaborations across the COVID-19 lockdown.

Throughout this article, we will refer to courses as an individual subject unit (also called modules) and programmes as the 3- or 4-year degree programme (called courses in some places) in which students enrol. Similarly, we will refer to Microsoft Teams in full and the student teams as groups to avoid confusion between teams and Microsoft Teams.

\section{METHODS}

Ethics approval was sought from The University of Nottingham, School of Biosciences ethics committee (SBREC190105A-R1).

\subsection{University and course context}

The documentary group assignment was implemented in the $3^{\text {rd }}$ year undergraduate course (module) Plants and the Soil Environment at the University of Nottingham, UK. The course runs in the spring semester (January-May) with a 3-hour contact block each week. In 2020 there were 24 students in the class from both the School of Biosciences (Faculty of Science) and the School of Life Sciences (Faculty of Medicine and Health Sciences; Figure 1). In addition to bridging two faculties, the students come from degree programs based at both our Sutton Bonington Campus, where the module is run (BSc Plant Science (13\%), BSc Agricultural science (17\%)) and our University Park Campus (BSc Environmental Science (37\%), BSc Environmental Biology (12\%) and BSc Biology (21\%; School of Life Sciences); Figure 1).

Plants and the Soil Environment explores how plants are adapted to different soil environments such as drought, flood, salinity, heavy metals, nutrient deficiency, and includes plant-plant interactions. In addition to exploring plants already adapted to these conditions, we discuss recent advances in crop breeding for improving food security under each of these conditions. The science learning outcomes are : Understand (a) the pathways and mechanisms involved in the uptake, transport and use of water and nutrients; (b) plant adaptive responses to water (drought and flood), nutrient, salt and heavy metal stresses; (c) crop improvement strategies based on the roots (biofortification, and pollutant exclusion/Safe crops); (d) have developed skills to evaluate root physiology (through field and computer sessions).

To achieve these concept objectives the course includes semi-traditional lectures for each of the themes (discussions are included in these lectures), a debate to explore the pros and cons of biofortification (facilitated by another staff member) and a computer lab on root image analysis techniques. In previous years the main assessments were two 1000-1500 word essays within two of the themes (of their choice) with an expectation of a deeper exploration of their chosen topics than is possible within the lecture time. However, students are also writing their dissertations during the spring semester and similarly at the end of semester there is a high marking load on staff from dissertations and exams. With this in mind the assessment was changed to a group work documentary video - still exploring a question of their interest within one of the themes to achieve the concept learning objectives of the course. Additional skill objectives were added: develop skills to (a) communicate complex ideas visually (in particular multimedia formats); and (b) to work in groups (supported by workshops and evidenced by a documentary video including a contribution statement in credits).

\subsection{Three aligned assessment tasks}

The assessment was designed around preparing a group-work documentary video exploring in depth a topic 
related to plant responses to soil environments. The first assessed coursework requires submission of a storyboard describing the flow of the documentary and a poster about the science to be covered (worth $20 \%$ of the course mark). These were designed to ensure feedback on the science content and flow of the videos with plenty of time for adjustments. Students presented the posters to the class and had group meetings with the lecturer (Rasmussen) to talk through their storyboard and receive feedback to maximise the quality of the end-product (documentary).

The second assessed coursework is the final video submission (10-15 minutes; worth 70\%). This was marked in two ways: 1) As a class the videos were viewed in the last session (including each staff member involved in the course, ensuring moderation of video marks); 2) Within the groups they rated each member's contribution. This second rating was to ensure each group member was held accountable for their work and was supported by training session 4 (see below). If a student was not contributing appropriately (and if these issues had been raised prior to the final submission) the marks would be scaled according to their contributions. In 2020 several groups discussed issues early and they were appropriately resolved resulting in all group members in each group receiving the same final marks. Marking rubrics (Supplementary Materials 1) were provided for both the poster (with an emphasis on science rather than artistic expression) and the video.

Finally, to ensure students had the opportunity to learn teamwork skills and strategies there were 4 compulsory tutorial sessions dedicated to the team assignment and team skill information (worth $2.5 \%$ each; details of sessions below) and were based on (Rasmussen et al., 2011)).

\subsection{Group allocations}

Students were asked to rank the 6 themes (salinity, heavy metals, flooding, drought, nutrients and plantplant interactions) in order of their top three choices. Students were then allocated to a theme based on these preferences and ensuring multiple degree programs were represented in each group. Multiple degree programs were important to maximise i) diversity of background knowledge; ii) the chance that not all students knew each other; and iii) inter-campus collaboration.

\subsection{Software: Microsoft Teams and Moodle Virtual Learning Environments}

Since the students came from two campuses, Microsoft Teams was used to facilitate their collaborations. Additionally, Microsoft Teams, and other collaborative software such as Slack, are now widely used in most workplaces, making Teams proficiency an important employability skill.

Prior to semester start, 6 Microsoft Teams environments were created, one for each theme. A class notebook (uses built-in OneNote) was set up in each Team. The collaboration space section was where all the student could see and add to the content. To partition the large amount of new information (such as how to keep meeting minutes, legalities of filming in public or conducting interviews and the risk assessment forms for students working off campus) subsections were made to contain each type of information (Figure 2). Once students were allocated to their Microsoft Team they added sections to the collaboration space to suit their own needs. Important files such as fliers to give the public, the risk assessment forms, a short tutorial on using Microsoft Teams (Supplementary Material 2) and PDFs of the 4 team training tutorials were all uploaded to the 'Files' area of each Team (Figure 3). As semester progressed the groups used the 'Posts' page to communicate with their other members and with the lecturer (Rasmussen) and had group meetings using the video call option.

Additionally, the University of Nottingham uses Moodle as a virtual learning environment. As such students also had access to all the lecture slides and recordings via Moodle and poster submission and team contribution forms were uploaded by each student to the assignment submission box by each deadline. The storyboard was left in the Microsoft Team environment and discussed during the lesson. The video files were uploaded to the class-wide Microsoft Team (created during semester to provide additional information about strike action and the COVID-19 advice).

For filming and editing footage, students used their own cameras or mobile devices and were provided with a series of links to open-source software. This was important as students have different mobile and computer 
devices so finding a flawless one-size-fits all is not trivial. However, there are plenty of options available and in the 'real world' they will most likely be making their own decisions on what software is most appropriate. Support was offered for trouble shooting if needed, however each group managed to successfully source and implement software.

\subsection{Team training}

The team training sessions were based on (Rasmussen et al., 2011). Briefly each session took around 30 minutes and were spread out at key times through semester. The first activity happened in week 2 when the students had just been allocated to a group and was an icebreaker, focused on expectations of team members, personality types and role/task allocation. Session 2 was based around negotiation and problem solving and used a negotiation activity. The third session had the students reflect on their behaviour in the team and that of the team as a whole (in-line with the expectations discussed in Session 1). As this third session occurred one week after moving to online teaching, the session was also used to think ahead about plans or behaviours that may need to change to overcome collaborating in isolation. The final session occurred in the last class before submission (via video call during lockdown) and had the students negotiate contributions to the final documentary product. Each session was available to the students from week 2 in their Microsoft Team environment as a PDF and with the text copied into the Collaboration Space in the Class Notebooks so they could answer questions and make notes. The student and teacher guidelines are available at https://www.arlab.co.uk/education-research.html (Supplementary Material 3). For a visual representation of the semester plan and when interruptions occurred, please see Figure 4.

\subsection{COVID-19}

Online teaching began at Nottingham on the $23^{\text {rd }}$ March 2020 (Figure 4). This came after the poster session and before the third team training session. There was just one lecture left which was put on Moodle. All the remaining sessions were tutorials and happened using video calls with each group within their Microsoft Teams. The class viewing was to be streamed using the whole class Microsoft Team. Unfortunately, due to stormy conditions the internet crashed halfway through the first video which was disappointing. However all but 2 students joined the non-compulsory session (one of those students was in a different time zone).

Aside from the unusual circumstances, and interviews needing to occur via video call, the course continued as programmed. Students were reminded that collaborations are often done with people in other countries or time-zones in much the same way they were working (they were also provided with links to YouTube videos of orchestras playing online as a Covid-19 special treat!).

\subsection{Data collection and analysis}

At the end of semester (after marks had been released), an anonymous Microsoft Forms survey was sent to all the students in the class (Supplementary Material 4 for the full set of questions). For comparison to previous team training experience (Rasmussen et al., 2011) the open questions regarding the benefits or detriments of teamwork were coded using the same keywords (Detriments: social/personal; conflict management; marks/assessment; logistics; unequal workload. Benefits: Learning; Shared workload; social/personal; enjoyable; marks; employability; and the only other response was about communications).

Specific to the COVID-19 lockdown situation, the open ended questions about the effects of COVID-19 were coded as positive (team support; improved logistics; adapting to the situation) or negative (Stress/loss of motivation; technical challenges such as editing or filming; communications/meeting in person). The data were plotted as percentage of total respondents (12), or as the number of responses (where students could choose more than one response or from the coded open-ended responses).

As this applies to only one cohort of 24 students and only 12 students responded to the anonymous survey, no additional statistical analysis has been conducted. Instead we provide quotes of student responses to further support our interpretations of the data. The full set of open responses can be found in Supplementary Material 5(A-E) 


\section{RESULTS}

Twelve of the 24 students in the class filled out the anonymous survey. The following results are based on their responses.

\subsection{Students generally have a positive attitude to teamwork.}

Of the 12 respondents, 10 reported positive attitudes (like 7; or love 3) to teamwork (Figure 5A). Positively, all students felt their teamwork experience in Plants and the Soil Environment (BIOS3015) was positive (ok 2; excellent 10; Figure 5B) and all students reported that their experience in this course was better than their previous experiences (Supplementary Material 6). The two respondents who mentioned that they dislike or hate teamwork both reported their experience in BIOS3015 was "Excellent" (Supplementary Material 5A).

In Plants and the Soil Environment (BIOS3015) none of the respondents reported conflict (Supplementary Material 5A) and when asked to explain, the majority of comments were about "worked well together" (IDs $1,4,8,9,11$ ) or "got along well" (IDs $5,10,11$ ). Some students made comments about how they maintained their positive relationships for example "All members equally divided the work and communicated effectively over WhatsApp group chat. By assigning different areas of research it allowed a bit of independent research as well, which helps in distance group work" (ID2) or "Nobody was particularly attached to their own ideas that they wouldn't allow other ideas" (ID3). Where issues arose students reported overcoming them "There were slight issues due to distance and lack of communication, however these were soon sorted" (ID7) and "Everyone got along; there were minimal disagreements and those that did arise were quickly resolved" (ID10). A respondent who loves teamwork reported: "My team was amazing we helped each either with respect and without fights" (ID6).

When allowed an open box to discuss detriments of teamwork, every comment fitted well within the codes used in our previous paper (Rasmussen et al., 2011). Five respondents mentioned unequal workloads as a problem while 4 students made comments linked to unfair marks (Figure 5C). Similarly, 4 students commented that it can be harder to organise meetings or other logistical issues while only 2 students commented on either conflict or social issues (Figure 5C). Logistical concerns were around making time to meet (Supplementary Material 5B; ID4 and ID9) and bringing the final pieces together (ID2) while conflict (reconciling differences) and social issues were closely related with different work paces (ID7), accepting your 'vision' may not be the outcome (ID3) and personality clashes (ID8 and ID10).

When describing the benefits of teamwork, all bar one comment fitted well within our previous codes (Rasmussen et al., 2011). This comment was linked to communication as a benefit. Although unequal workload was the most mentioned detriment, shared workload was also one of the most mentioned benefit of teamwork (Figure 5D). Benefits to learning were also mentioned and included comments about everyone having different skills and background knowledge. Two comments were made about social benefits and for each of 'Enjoyable', 'marks', 'employability' and 'communications' there was one response. On inspecting the comments that fall in the Learning and Shared workload categories, there are many similarities (Supplementary Material 5B). For example, several students refer to pooling ideas, strengths or skills (IDs 1,2,4,6,7,8,12). As mentioned by respondent ID10 "The end result is often "greater than the sum of its parts", less work has to be done overall, for a bigger result".

\subsection{Students see structured team training as useful.}

To determine which components of the course are important as seen by the students we asked them to select which activities through semester they found useful and why (Figure 6A). We also asked them what they would do differently in future team experiences (Figure 6B); how much they used different aspects of Microsoft Teams (Figure 6C) and whether they used any additional software (Figure 6D).

Even though this is a third-year course, of the four Team Training sessions (which were all compulsory) the first session (about getting to know your team) was chosen as useful by 11/12 respondents. Half the respondents selected Team Training Session 3 (reflection on progress) and 5 selected Team Training Session 4 (negotiating author order) as useful. Even though the students said they experienced no conflict 
(Supplementary Material 5A) a quarter of the respondents still chose Team Training Session 2 (negotiation tips) as useful. These responses demonstrate that each session is considered useful to at least a third of the respondents (Figure 6A).

During week 2 (when the first Team Training session was run) the students were also given a tutorial on using Microsoft Teams. Even though the Microsoft Teams platform was having technical difficulties and only returned as the class ended, 9 of the 12 respondents still said this session was useful. They were provided with a handout with screenshots to help them (Supplementary Material 2). Similarly, the poster session with feedback was considered useful by 8 of the respondents (Figure $6 \mathrm{~A}$ ).

Interestingly 11 of the 12 respondents felt that having the sessions compulsory was useful (Figure 5A) Reasons for this included: "... ensured all the team would try to be present" (ID1) "..ensured we had deadlines in the back of our heard throughout the process" (ID2), "... made it easier to organise group meetings" (ID5), "... necessary to keep everyone on track" (ID8). These indicate that even though the reason these sessions were made compulsory was to ensure everyone did the training, the students found them important for 'ensuring' in-person meetings (Supplementary Material 5C).

Positively, the reasons given for why the reflection activity was useful ["Team reflection gave chance for people who were lacking behind to pull their weight" (ID5), "Team reflection allowed us to see where we stood and what we need to work on still" (ID2; Supplementary Material 5C)] - are the goals that the activity was designed to meet. One student commented that Team Training Session 3 (reflection activity) "came too early for our group" (ID7) - however the tasks described actually refer to the fourth session (discussing contributions). This confusion possibly came about because they were reminded during the third session that they would be discussing contributions in the final session.

A few students also commented on sessions they felt were less useful - in particular negotiation (Team Training Session 2): "The negotiation session I found to have its heart in the right place but didn't feel very effective. I think it was because we didn't always believe the arguments we were putting forward" (ID10), "our team worked well together so didn't need negotiation session or reflection" (ID11). In future additional tasks (for example getting the students to think about something they may have disagreed on in the assessment and discuss how they came to a solution) may help widen the benefit of that session in particular. However, a third of the respondents still felt the session was useful (Figure 6A).

For comparison to our previous research (Rasmussen et al., 2011) we used the same codes for open responses to what they would do differently in their next team experience (Supplementary Material 5D). All the responses fell within these categories with 8 references to behaviours around group organisation such as "Add each other on social media" (ID4), "Always set small goals and keep the team updated" (ID6), "Plan more and start earlier on" (ID12). Workload division, ways to approach tasks and problems; and personality traits were also mentioned - mostly in the context of the first Team Training Session. For example: "Discuss early the strengths and weaknesses of the team members, and the expectations of how the team and the members will perform both alone and together." (ID7); "Division of labour earlier on" (ID8); "be organised from the start about delegating work to team members (ID11; Supplementary Table 5D). Of particular note was a comment about personal behaviour which demonstrates a very mature attitude "Check-up quietly with other group member just to see if they are doing okay or if they need support with the workload" (ID1). This behaviour is mentioned in the first and third Team Training Sessions, however the COVID-19 situation made this a necessary behaviour in several groups and highlighted how problems can be dissolved before there is friction with an informal friendly (non-task-related) chat.

When asked about which features of Microsoft Teams the students used the most, unsurprisingly uploading files was used the most (they also had to upload the final video in this way). Perhaps more surprising was that only half the respondents said they used the Posts function (text chats that everyone can see) a lot or all the time (Figure 6B) and only 3 used the Microsoft Teams mobile app a lot or all the time (Figure 6B). This may be because 6 also mentioned they used WhatsApp or Facebook Messenger (Figure 6C).

\subsection{Using hybrid learning strategies is more resilient to disruptions such as COVID-19}


Since this was the first time this task had been applied to this course, the students were asked if there was anything that made it difficult to complete the assignment (Figure 7A). Not surprisingly 5 of the 9 challenges mentioned by students in the open box were specifically relating to COVID-19. Of those 5 responses, 4 were about the difficulty of collaborating in lockdown because meetings were now more difficult (2) or because viewing or working together on visual aspects of the assessment were more difficult (2) (Figure 7A). The general comment was unspecific "Covid issues were the only detrimental issues." (ID7).

Not specifically related to COVID-19, two students mentioned difficulties with Microsoft Teams (one relating to Teams crashing, the other to the time taken to upload and share files) and one student mentioned difficulties editing (Figure 7A; Supplementary Material 5D). One student said there were no difficulties and two declined to describe any difficulties.

To identify the main impacts of COVID-19 on their learning experience they were asked how much the course (Plants and the Soil Environment, BIOS3015) was affected by COVID-19 compared to their other courses (Figure 7B). All except one respondent said that their other courses were affected a lot by lockdown, and the one student said their other courses were affected a little. In contrast only 4 students said COVID-19 affected BIOS3015 a lot, 4 said BIOS3015 was affected a little and 4 said it was 'not much' affected.

The open explanations of how COVID-19 affected their assessment in BIOS3015 were coded into negative (9 points total) or positive ( 7 points total) impacts. The negative impacts in particular were around the difficulty with arranging meetings and communicating during lockdown (Figure 7C). Other issues were around the difficulty editing or filming in lockdown and loss of motivation or higher stress levels. Slightly surprisingly were the number of neutral or positive responses where students commented that they had their team support, or it was good to adapt to the situation (Figure 7D). Two comments were also related to less time spent in transit or more efficient meetings via video (Improved Logistics; Figure 7D).

\subsection{Additional comments}

The final question on the survey was an open-ended question box where students were invited to provide any additional comments about teamwork or the course. Only three students commented here and all were positive. Respondents 7 and 9 both provided comment on issues/improvements. For example: "a shame that we were unable to have the popcorn session, and the lack of poster feedback from the class was disappointing" (ID7). The 'popcorn session' was to be an optional viewing session in the last programmed class and 23/24 students logged into the BIOS3015 class team to join in. Unfortunately a storm took out the internet in our local area where several lecturers lived, resulting in the session being cancelled. Also the poster feedback happened online rather than the planned in-person session, so fewer students engaged with providing the feedback. In future this session will be done as a class (preferably in the classroom but could also be in the BIOS3015 Class Microsoft Team environment) with instant feedback given in the chats.

The other potential 'improvement' suggested was a shorter video and an exam based on all the content. The reason just one topic is requested of each group is so they can explore the science in depth, linking scales and different physiological and genetic responses. Since the general processes apply similarly across many of the stresses explored, by understanding one in detail students can then apply this to any future situation. Memorising the content of every lecture for an exam would not achieve this goal. However, in future it may help to make this overarching goal clearer to the students in the course objectives and reminders provided throughout the semester.

\section{DISCUSSION}

Collectively the results presented here demonstrate that the semester-long group video assignment blending synchronous and asynchronous online and in-person teaching tools was perceived as a positive experience by the students. From every respondent reporting that this group experience was better than their previous experience, to detailed comments such as "My team was amazing we helped each either with respect and without fights" and "This experience has made me less terrified of group work, and hope that all my future teams are this good". Most impressively these responses come in spite of the difficulties associated with 
collaborating during COVID-19-induced national lockdown (and in some cases international travel to home countries).

Although we have used the term 'group' to refer to the student teams here to avoid confusion with Microsoft Teams, the student responses illustrate their appreciation of the interdependencies associated with effective team functioning. This includes exploiting the individual expertise that each member brought to the team and supporting each other during lockdown.

\subsection{Team training}

Positively this study demonstrates that the team training activities designed for an Australian $1^{\text {st }}$ year biology course (Rasmussen et al., 2011) was also effective in a British $3^{\text {rd }}$ year plant science course and allows for comparisons between studies (same team training sessions run by the same lecturer).

Interestingly there was a large difference in student perceptions of teamwork. While only $59 \%$ of first year students reported a positive attitude to team-work (Rasmussen et al., 2011), here $84 \%$ of our third year students reported positivity towards teamwork (and all had a positive experience in this team assignment). Despite this disparity the reasons for liking teamwork were similar with Learning/academic and shared workload/working as a team as the two most reported benefits in both studies followed by social/personal reasons. Similarly Wilson et al., (2018) found meeting new people $(71 \%)$, sharing workload $(67 \%)$, getting to work with friends (56\%), Peer teaching experiences (54\%) as the most common team-work benefits in science undergraduates (Wilson et al., 2018). However they found less than half their respondents reported positive teamwork experiences and less than $20 \%$ believed they achieved a better mark than if they worked individually (Wilson et al., 2018).

Unsurprisingly with their honours dissertations (in addition to other courses) being concluded parallel to this team assignment, our third-year students were less concerned with social or personal benefits of teamwork compared to the first year students (Rasmussen et al., 2011). While unequal workload was the most common detriment of teamwork in both studies, our third year students reported logistics and marks as more concerning than conflict or social issues which were both important to the first year cohort (Rasmussen et al., 2011). These seem to be common across institutions, year groups and science disciplines as scheduling meetings (logistics), unequal workload, relying on others, time management and conflict were also the most common detriments reported in (Wilson et al., 2018).

Including social interactions and an introduction to logistics, as implemented in the first Team Training Session, are important for encouraging the development of team synergies and a shared understanding of the task goals (Panteli et al., 2019). This will be equally important in virtual teams to overcome that sense of 'working with strangers' (Panteli et al., 2019). In contrast to our study, plan-making activities were only found to improve engagement early in semester (Kizilcec et al., 2020). One reason for this difference may be because students were not asked to follow up and reflect on their plan implementation and working as individuals (Kizilcec et al., 2020) there was less incentive to stick to a plan. In contrast our students were asked to reflect on their actions in Session 3 (using the same list of behaviours introduced in the first session) and working in teams where they were held accountable for their contribution encourages more consistent engagement.

There were also differences between cohorts in relation to the usefulness of each Team Training Session. In both BIOL1030 (Rasmussen et al., 2011) and Plants and the Soil Environment only a third of the respondents found the second Session (negotiation) useful. Interestingly while only $40 \%$ of the first year students found the first session (team forming) useful, $92 \%$ of the third years reported that it was useful with reasons such as "helped to establish people's strengths and weaknesses" and "Great to have time to meet people that you are working with but might not know". This emphasises that in diverse courses (and in programmes where some students spend a year in industry between second and third year) the students even at third year may not know each other.

While negotiating contribution was considered useful by the most students in the first year course (Rasmussen 
et al., 2011), it was the second least useful according to the third years. This may reflect that the third year students 'leant' on each other more for support as a result of lockdown and reported fewer conflicts (none reported in this class while more than a quarter reported team-conflict-related issues in the first years (Rasmussen et al., 2011)). It is also relevant to note that sharing emotions and feelings can help build camaraderie in a team (Ayoko et al., 2012). The exceptional circumstances created by COVID-19 may have forced our students into a situation where they felt it was acceptable to talk about how they were which may have helped move all the teams into a 'performing' state where they worked together to overcome complex problems. Similarly it may be that the training was enough to enable the students to overcome (at least smaller) conflicts before they became a problem using the non-personal mediation-style techniques. Ayoko et al., (2012) reported that teams moving into 'storming' tended to resort to personal attacks, which can damage self-confidence. However they noted that the successful teams used feedback-seeking or mediation behaviours (Ayoko et al., 2012) - the type of exercises included in our Team Training Session 2.

Our students also reported that ensuring the sessions were compulsory was important for regular team contact. Other studies have also reported that students appreciate regular allocated class time for teamwork (Wilson et al., 2018) and Gleadow et al., (2015) found that blog engagement increased when marks were also allocated for comments - further evidence that compulsory sessions or components need to be associated with marks to ensure success.

Since the marking criteria were presented as explicit rubrics with different expectations (and learning objectives) to the previous year's essays it is unhelpful to compare the marks between cohorts. However it is useful to note that the video groups performed better than expected based on the rubric provided and expectations will be raised for the 2020-2021 cohort. Other studies have also found that multimedia activities are often more explicit in their expectations leading to improved learning outcomes (Mercer-Mapstone \& Kuchel, 2015). Additionally when those multimedia tasks involve communicating to a non-science audience (as in our task) students also had a better understanding of the content (Mercer-Mapstone \& Kuchel, 2015).

As highlighted by Andrew Churches (2008), videos and animation (implemented by several teams) are tasks creating knowledge (top of Blooms (digital) taxonomy). Videos are also good for encouraging team interaction, providing a multimodal educational experience (Visosevic \& Myers, 2017). This paper is not focussed on the video as a mode of education but rather uses it as an example of an appropriate team assignment that incorporated explicit team skills and online collaboration training. For more detailed analyses of multimedia assessments see (Kuchel et al., 2014; Visosevic \& Myers, 2017).

While COVID-19 was an extreme disruption, our students overcame the challenge, becoming cohesive teams and producing decent end products (videos). They even enjoyed the task and were engaged such that they all (except 2) logged in to watch online together in the last (non-compulsory) session. This raises an interesting (and potentially controversial) question about whether inflicting some controlled disruption may in fact better prepare our graduates for a changing world. By providing them with the tools to succeed they not only had a positive team experience but gained confidence for future challenges.

\subsection{Hybrid learning environments}

Use of social media like Facebook (Chu et al., 2019) and SnapChat (Faulkner, 2018) have previously been shown to be effective online collaboration spaces because many students are already familiar with the platform for personal use and unlike many of the online education platforms (like Moodle) these social media forms have apps for mobile devices allowing them to engage/interact anywhere, anytime (Chen \& Chiou, 2014; Chu et al., 2019; Gleadow et al., 2015; Singh, 2017). The recent advancement of collaborative platforms like Microsoft Teams (used here) has included the development of accessible apps capturing this anytime, anywhere flexibility that can be used for enhanced learning environments using professional platforms (and university usernames).

Hybrid classes have been reported to have a stronger sense of community than those in traditional classrooms (Chen \& Chiou, 2014; Singh, 2017). Once students see themselves as belonging to a group, they feel trust and comfort in the community (Chen \& Chiou, 2014), a possible explanation for why our students responded 
within their own small teams to comments/questions posted on the whole class Team. In our class, the sense of belonging is likely to be enhanced be a 'shared suffering' from lockdown, a sense that 'we're all in this together'. Similarly, using video calls from their homes may have further strengthened the sense of belonging. It has also been suggested that the convenience of hybrid methods alone are enough to increase the ease of interactions (Chen \& Chiou, 2014).

Although some studies have found that learning is improved and higher grades obtained in hybrid learning environments (Chen \& Chiou, 2014; McFarlin, 2008; Singh, 2017), it is very important to keep in mind that context is important and that diversity across courses is important. Kizilcec et al., (2020) found that scaling behavioural science interventions across many learning contexts can dramatically reduce their effectiveness. So while the video task presented here has been demonstrated to work across first and third year students in two countries, we are not suggesting these same strategies be applied to multiple levels within a single degree program. It is still important that students have training (via assessments) in more traditional tasks like scientific writing, posters and PowerPoint presentations. We predict that mixing these with nontraditional tasks and embedding the tasks within online collaborative spaces like Microsoft Teams will have the greatest benefit to the most students. To achieve this via resilient and inclusive methods, horizon scanning is important (Gleadow et al., 2015; McFarlin, 2008). No-one could have foreseen the COVID-19 disruptions, however using Microsoft Teams from the beginning of semester, despite the challenges of being an early adopter, created a resilient learning environment that withstood floods and pandemic.

Our findings that explicit training of team skills (including virtual collaboration) enhances the student experience is also supported by wider studies of communication skills (Mercer-Mapstone \& Kuchel, 2016). They also make the point that explicit interventions will only be successful if sustainable (Mercer-Mapstone \& Kuchel, 2016) which depends on the academic acknowledging the importance of the skill to student learning and the ease of implementation (Mercer-Mapstone \& Kuchel, 2016). The team training exercise here has been designed for ease of implementation, centred on 4 team training sessions that take no more than 30 minutes each (https://www.arlab.co.uk/teaching-teams.html). Additional scaffolding was provided via instructions on how to use Microsoft Teams (Supplementary Material S2) and in-class time was allocated to self-directed team assignment work (in the presence of the academic) so any issues could be easily resolved. The unusual COVID-19 educational circumstances have demonstrated that this scaffolding is adequate to ensure students have the tools to succeed in a virtual team environment.

\subsection{Enabling resilience: 5 key points}

The ease with which we moved to online-only teaching at the onset of lockdown is likely due to the following key points:

1) Microsoft Teams training: The students were already familiar with working in Microsoft Teams from week 2 of the semester.

2) Team cohesion: Students had already interacted in person during classes and the first 2 compulsory team training exercises. This meant they had already developed a rapport making the online interactions less daunting. This can be done online too, but it's important to facilitate the interaction in that first team session with video calls.

3) Reflection: The third compulsory team training session happened one week after lockdown which meant not only did students reflect on how things had been so far, but were also able to discuss what new challenges they might be facing and get support from the lecturer where needed (for example a lesson on screen-sharing was a common request).

4) Aligned (and frequent) assessment: the poster session was designed for early in semester to ensure students begin work on the research early in semester rather than leaving everything until the Easter break (which happened with the essays in previous years). The compulsory sessions spread through semester were worth a total of $10 \%$ and were directly linked to their teams (and timed appropriately - see above). The mark allocation/compulsory status of these meant students actively engaged with their team throughout semester. 
The students themselves noted how important these compulsory sessions were. The video was worth the most with students also asked to provide a report on each team members contribution - ensuring student accountability.

5) Teaching Adaptability: importantly regular contact (in-person or virtual) between students and with lecturer meant problems and challenges could be adapted as needed starting with the minor flood interruption, and more significantly facilitating student team communication after COVID-19 lockdown.

Finally, a separate point is that the fourth session is a representation of academic negotiation - deciding paper authorship order (many students do not realise this organisation until they do this fourth session). Similarly, co-authors provide an author contribution statement at the end of each manuscript as commonly required by most journals. As such, Session 4 not only ensures students are held accountable for their marks, but also links the video communication task back to a real-world research scenario - the pride of research-intensive universities.

\subsection{Enabling resilience: a few adjustments!}

Context is key! As this was the first year this learning strategy was implemented for this course there are a few adjustments to be made to further enhance flexible student learning.

Session implementation: If the entire program runs in a virtual environment the compulsory sessions do need to include a discussion with the lecturer. Setting up a schedule for exactly when each team would meet with the lecturer is important to ensure everyone is online in time and it then becomes a focal time for further discussion without the lecturer (often both before and after the lecturer-mediated conversation).

Session 1: The first session will be most important as reported by the students - for getting to know each other and decide what tasks each person will be the lead on based on personalities and existing skill sets. In a virtual environment this may be more awkward and therefore teacher support and prompts may be more necessary than it was in the classroom.

Session 2: More advanced negotiation tasks using role play. These could be created as hypothetical situations for common problems (like social loafing). Similarly, this session would be useful to remind students that expression of emotion is not bad and can help identify problems. To avoid the discussion becoming personal (conflict) use invitations for feedback so everyone becomes more open to discussion (Ayoko et al., 2012).

Session 3: In addition to the reflection task (which is done individually) it would be useful to add an activity where students discuss in their teams what their personal motivations are for the task - what do they want to gain from the task/module. This type of task has been found to increase motivation and completion particularly for marginalised students (Kizilcec et al., 2020). In a team setting this could help remind everyone that they have common goals while further strengthening the cohesiveness of the group.

\section{ADDITIONAL RESOURCES}

Link to course training materials (team training sessions (student and teacher documents), and Microsoft Teams tutorial handout and videos): https://www.arlab.co.uk/teaching-teams.html

Link to public videos from BIOS3015 Plants and the Soil Environment : https://mediaspace.nottingham.ac.uk/category/Plants\%20and\%20the\%20Soil\%20Environment/166633361

Link to Podcast about this assessment (Episode 9): https://digitaleducationpractices.com/podcast/

\section{LITERATURE CITED}

Allan, E. G. (2016). "I Hate Group Work!": Addressing Students' Concerns about Small-Group Learning. InSight: A Journal of Scholarly Teaching, 11, 81-89.

Ayoko, O. B., Konrad, A. M., \& Boyle, M. V. (2012). Online work: Managing conflict and emotions for performance in virtual teams.European Management Journal, 30 (2), 156-174. doi:https://doi.org/10.1016/j.emj.2011.10.001 
Chen, B. H., \& Chiou, H.-H. (2014). Learning style, sense of community and learning effectiveness in hybrid learning environment.Interactive Learning Environments, 22 (4), 485-496. doi:10.1080/10494820.2012.680971

Chu, S. K. W., Ravana, S. D., Mok, S. S. W., \& Chan, R. C. H. (2019). Behavior, perceptions and learning experience of undergraduates using social technologies during internship. Educational Technology Research and Development, 67 (4), 881-906. doi:10.1007/s11423-018-9638-2

Churches, A. (2008). Bloom's Digital Taxonomy . Retrieved from http://burtonslifelearning.pbworks.com/f/BloomDigitalTaxonomy2001.pdf

Faulkner, S. (2018). Top 10 reasons your students will thank you for using Snapchat as a tutorial tool. Retrieved from https://suzannessnapchat.wordpress.com/2018/02/27/top-10-reasons-your-studentswill-thank-you-for-using-snapchat-as-a-tutorial-tool/

Garcia, M. G., Lopez, C. B., Molina, E. C., Casas, E. E., \& Morales, Y. A. R. (2016). Development and evaluation of the team work skill in university contexts. Are virtual environments effective? International Journal of Educational Technology in Higher Education, 13 (1). doi:10.1186/s41239-016-0014-1

Gillespie, J. (2012). Enhancing Social Work Education Through Team-Based Learning. Journal of Social Work Education, 48 (2), 377-387. doi:10.5175/JSWE.2012.200900014

Gleadow, R., Honeydew, M., Ford, A., Isaac, B., \& Abbott, K. (2015). New tools for a new age: An evolution or revolution in higher education? F1000Research, 4 , 1502. doi:10.12688/f1000research.7533.1

Jimenez, A., Boehe, D. M., Taras, V., \& Caprar, D. V. (2017). Working Across Boundaries: Current and Future Perspectives on Global Virtual Teams. Journal of International Management, 23 (4), 341-349. doi:https://doi.org/10.1016/j.intman.2017.05.001

Kizilcec, R. F., Reich, J., Yeomans, M., Dann, C., Brunskill, E., Lopez, G., . . . Tingley, D. (2020). Scaling up behavioral science interventions in online education. Proceedings of the National Academy of Sciences, 117 (26), 14900-14905. doi:10.1073/pnas.1921417117

Kuchel, L. J., Stevens, S. K., Wilson, R., \& Cokley, J. (2014). A documentary video assignment to enhance learning in large first-year science classes. International Journal of Innovation in Science and Mathematics Education, 22 (4), 48-64.

McFarlin, B. K. (2008). Hybrid Lecture-Online Format Increases Student Grades in an Undergraduate Exercise Physiology Course at a Large Urban University. Advances in Physiology Education, 32 (1), 86-91.

Mercer-Mapstone, L., \& Kuchel, L. (2015). Teaching Scientists to Communicate: Evidence-based assessment for undergraduate science education. International Journal of Science Education, 37 (10), 1613-1638. doi:10.1080/09500693.2015.1045959

Mercer-Mapstone, L. D., \& Kuchel, L. J. (2016). Integrating Communication Skills into Undergraduate Science Degrees: A Practical and Evidence-Based Approach. Teaching \& Learning Inquiry: The ISSOTL Journal, 4 (2). doi:10.20343/teachlearninqu.4.2.11

Panteli, N., Yalabik, Z. Y., \& Rapti, A. (2019). Fostering work engagement in geographically-dispersed and asynchronous virtual teams.Information Technology 83 People, 32 (1), 2-17. doi:10.1108/itp-04-2017-0133

Rasmussen, A., Rossini, R., \& Kuchel, L. (2011). Is it worth taking time out of first year science courses to explicitly teach team skills? Paper presented at the 34th HERDSA Annual International Conference, Gold Coast, QLD Australia. Fully published paper retrieved from

Singh, J. (2017). Enhancing Student Success in Health Care Programs: Active Learning in a Hybrid Format. Journal of Instructional Pedagogies, 18 . 
Visosevic, T., \& Myers, A. (2017). Video Essay: the multimedia assignment of now. Paper presented at the Proceedings of the 2nd Association for Visual Pedagogy Conference, At Aalborg University, Denmark.

Wilson, L., Ho, S., \& Brookes, R. H. (2018). Student perceptions of teamwork within assessment tasks in undergraduate science degrees.Assessment $\&$ Evaluation in Higher Education, 43 (5), 786-799. doi:10.1080/02602938.2017.1409334

Supplementary Material:

S1: Marking rubrics

S2: Microsoft Teams tutorial

S3: Link to Team Training package - on my webpage https://www.arlab.co.uk/education-research.html

S4: Evaluation questions

S5: Evaluation open ended responses

S6: All students felt it was better than previous experiences

Figure Legends

Figure 1: Degree programs for students taking Plants and the Soil Environment in spring 2020. Total number of students in the class was 24. BSc Biology degree programme is based in the School of Life Sciences (LS), Faculty of Medicine and Health Sciences while all the other degree programs are within the School of Biosciences, Faculty of Science. Biology, Environmental Science and Environmental Biology are based at our University Park Campus while Plant and Agriculture sciences are based at our Sutton Bonington campus. The module is run on the Sutton Bonington Campus.

Figure 2: Class Notebook within each of the 6 Microsoft Teams environments. Subsections set up within the Collaboration space are visible and can be edited by all members of the team.

Figure 3: Files uploaded within each of the 6 Microsoft Teams environments.

Figure 4: Semester planning. The first week of semester was $27^{\text {th }}$ January 2020, each Monday we had a 3-hour teaching block which contained lectures (green) workshops (lighter blues) or programmed team time (dark blue), spring break is 4 weeks based around Easter (yellow). Assessments included compulsory team training sessions (2.5\% each), poster and storyboard (20\%), and the final video submission (70\%). The semester was interrupted by flooding, strike action (mostly impacting nutrient lectures), and COVID-19.

Figure 5: Student perspectives of teamwork. Percentage of respondents who love, like, dislike or hate team work (A); Students had ok or excellent team experiences in Plants and the Soil Environment (BIOS3015; B); Percentage of respondents that mentioned the listed detriments (C) or benefits (D) of team work as coded from open ended responses. $\mathrm{N}=12$ respondents.

Figure 6: Student perspectives of structured team training program and use of collaborative technologies. (A) Number of respondents finding different aspects of the course useful: first four activities were explicit team training workshops, 'Using Microsoft Teams' was a tutorial on how to use the online collaboration software, the poster session was assessed and student were provided feedback on science content and video plan and the final point was the number of respondents who felt having 'Compulsory Sessions' was useful. B) Coded open-ended responses for what students would do differently in their next team experience. C) the amount that students used different Features within Microsoft Teams: 'MT (Microsoft Teams) mobile' application, 'Collaboration space' was within the built-in OneNote Class Notebook addin, 'Files' is the page where students can upload and share files and 'Posts' is where students can have text chat conversations (or video calls) with the other member of their Microsoft Team. (D) visual representation of the other collaboration technologies mentioned by students in an open-ended question - The size of each rectangle represents the proportion of responses allocated to that feature, the number of respondents is given in the 
lower left of each box, FB = Facebook Messenger. Total respondents $=12$; for $\mathrm{B}$ and $\mathrm{D}$ the total does not equal 12 because students may have mentioned more than one point in their response.

Figure 7: Student perspectives of COVID-19 challenges. When asked about any difficulties they faced completing the assignment, most comments were related to COVID-19 (A). The size of the boxes (A) represent the proportion of responses in that code category, the number of responses is given after each code name. B) Respondents perception of the impact of COVID-19 on Plants and the Soil Environment (BIOS3015) or their other courses. (C) the number of comments considered negative impacts of COVID-19 on their assignment for BIOS3015 and (D) the number of comments considered neutral or positive impacts of COVID-19 on their assignment. Total respondents $=12$; for A,C,D the total does not equal 12 because students may have mentioned more than one point in their response.

Data Accessibility Statement

All data from this study are available within the main figures and supplementary material. All additional resources are available on the author's website: https://www.arlab.co.uk/teaching-teams.html. As in line with our ethics approval all data has been deidentified and contains no personal information.

Competing interests statement

The authors have no conflicts of interest.

Author Contributions

AR designed and implemented the activities, collected survey results and wrote the manuscript. QW provided technology support with MS Teams, helped with data analysis and draft editing.

Acknowledgements

AR would also like to thank Louise Kuchel (University of Queensland) for ongoing discussions and support with the original team training implementation in BIOL1030. Similarly, AR would like to thank Robby Wilson (University of Queensland) for the inspiration of video assignments. At the University of Nottingham, AR would like to thank Fiona Mccullough for her support with fitting a non-traditional task within existing student support frameworks and Kate Millar for support with ethics questions. Finally, AR would like to thank Martin Broadley, Darren Wells, Diriba Kumsa, Neil Graham and Andrew French for providing their expertise to the module and support with marking the videos. 


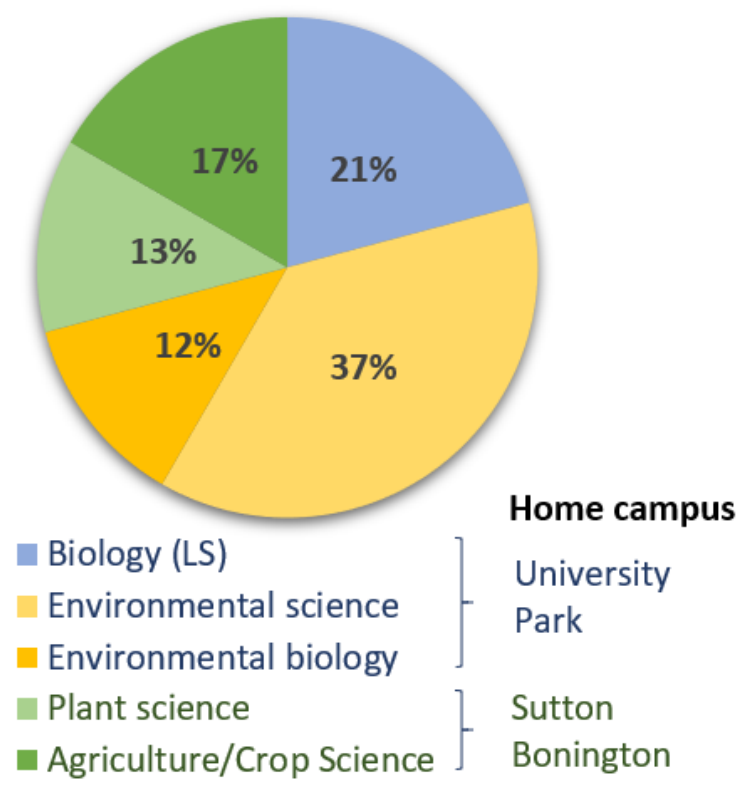

Figure 1: Degree programs for students taking Plants and the Soil Environment in spring 2020. Total number of students in the class was 24. BSc Biology degree programme is based in the School of Life Sciences (LS), Faculty of Medicine and Health Sciences while all the other degree programs are within the School of Biosciences, Faculty of Science. Biology,

Environmental Science and Environmental Biology are based at our University Park Campus while Plant and Agriculture sciences are based at our Sutton Bonington campus. The module is run on the Sutton Bonington Campus. 


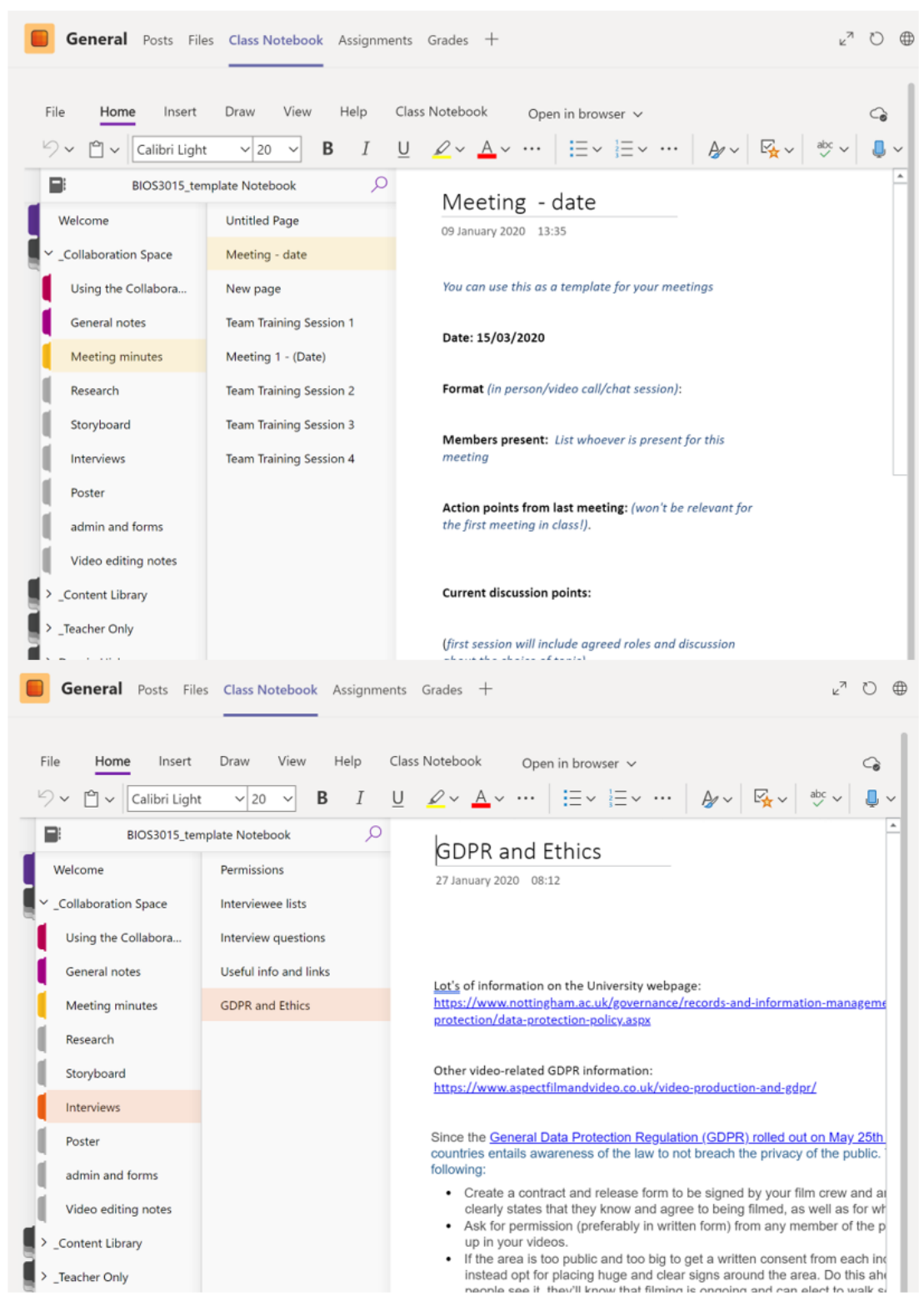

Figure 2: Class Notebook within each of the 6 Microsoft Teams environments. Subsections set up within the Collaboration space are visible and can be edited by all members of the team. 


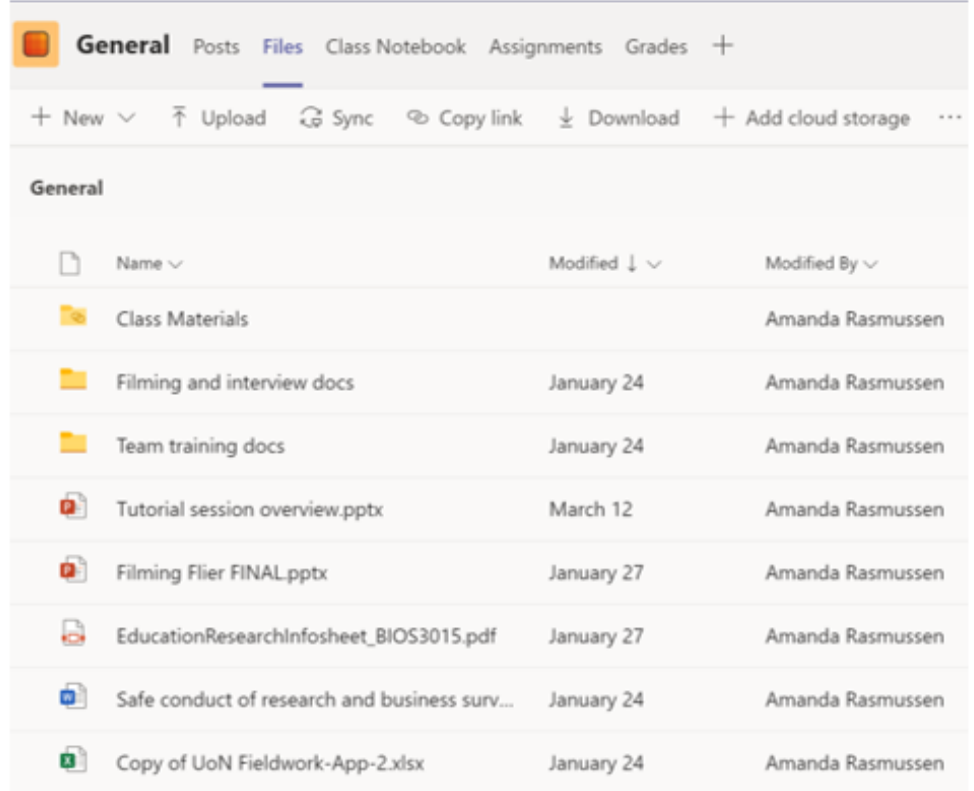

Figure 3: Files uploaded within each of the 6 Microsoft Teams environments. 


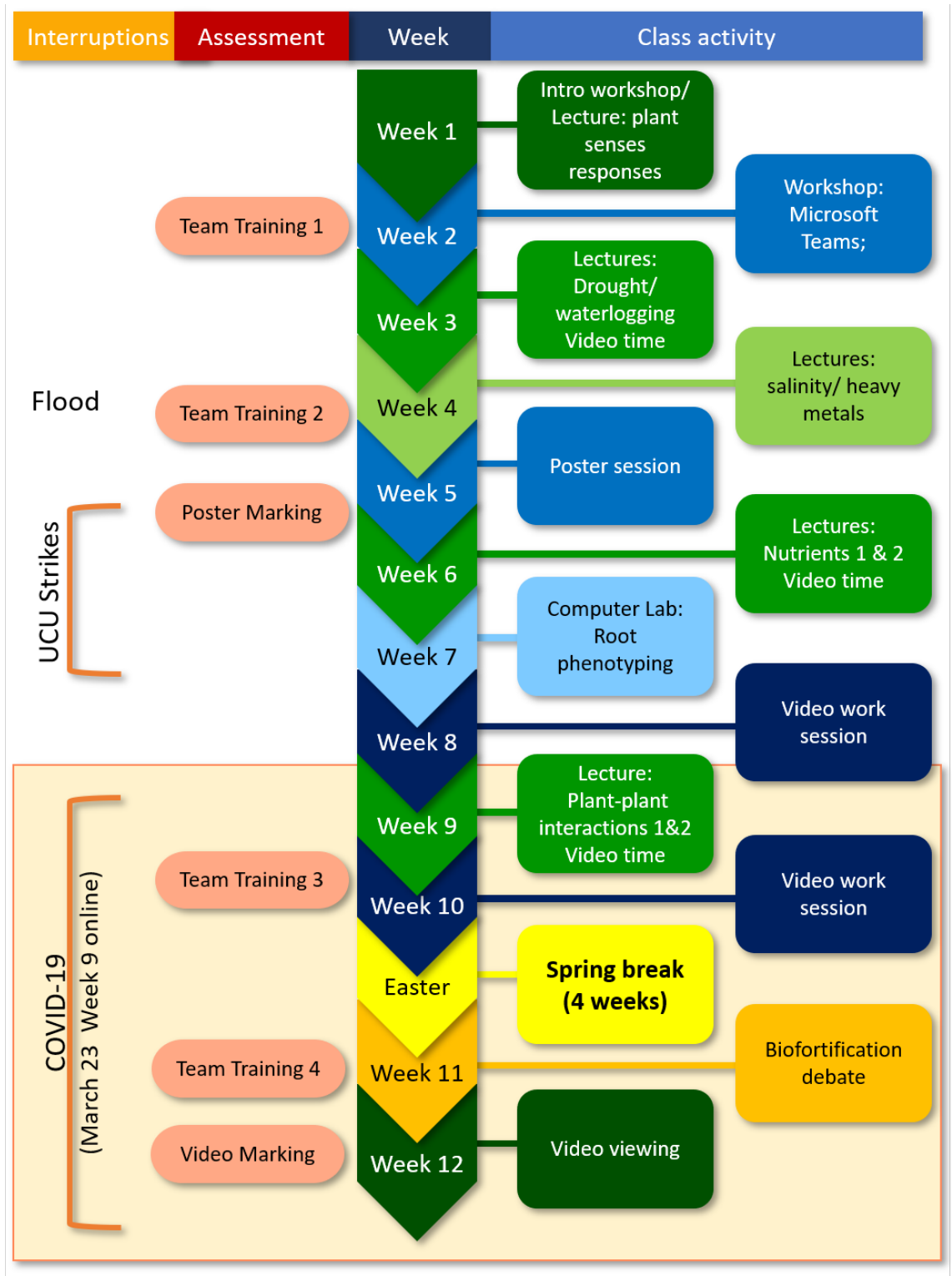

Figure 4: Semester planning. The first week of semester was $27^{\text {th }}$ January 2020, each Monday we had a 3 hour teaching block which contained lectures (green) workshops (lighter blues) or programmed team time (dark blue), spring break is 4 weeks based around Easter (yellow). Assessments included compulsory team training sessions (2.5\% each), poster and storyboard (20\%), and the final video submission (70\%). The semester was interrupted by flooding, strike action (mostly impacting nutrient lectures), and COVID-19. 
A

Attitude to teamwork

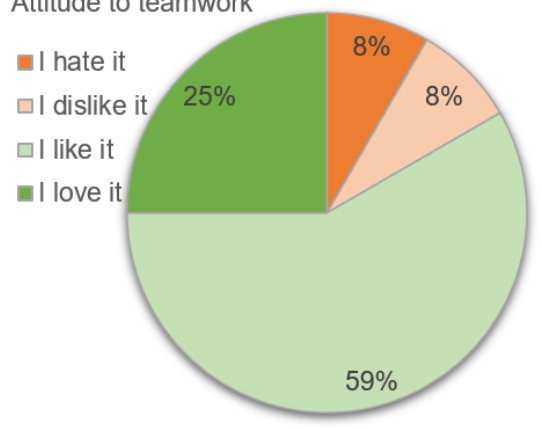

C

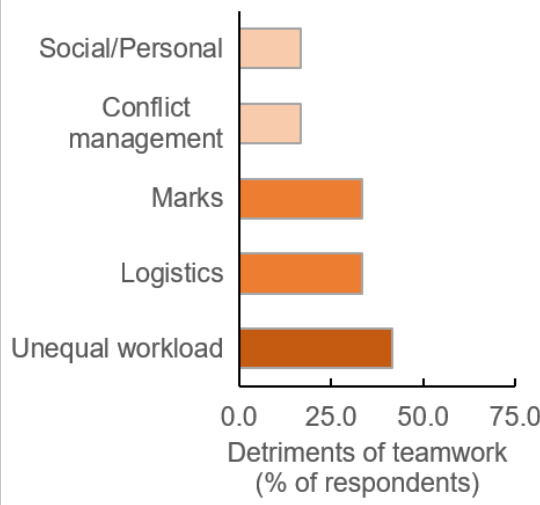

B

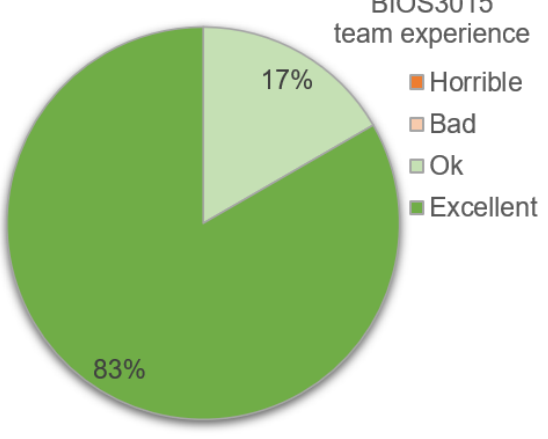

D

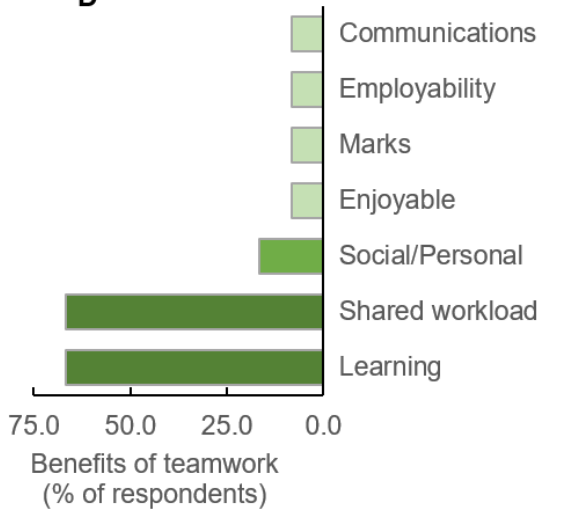

Figure 5: Student perspectives of teamwork. Percentage of respondents who love, like, dislike or hate team work (A); Students had ok or excellent team experiences in Plants and the Soil Environment (BIOS3015; B); Percentage of respondents that mentioned the listed detriments (C) or benefits (D) of team work as coded from open ended responses. $\mathrm{N}=12$ respondents. 
A

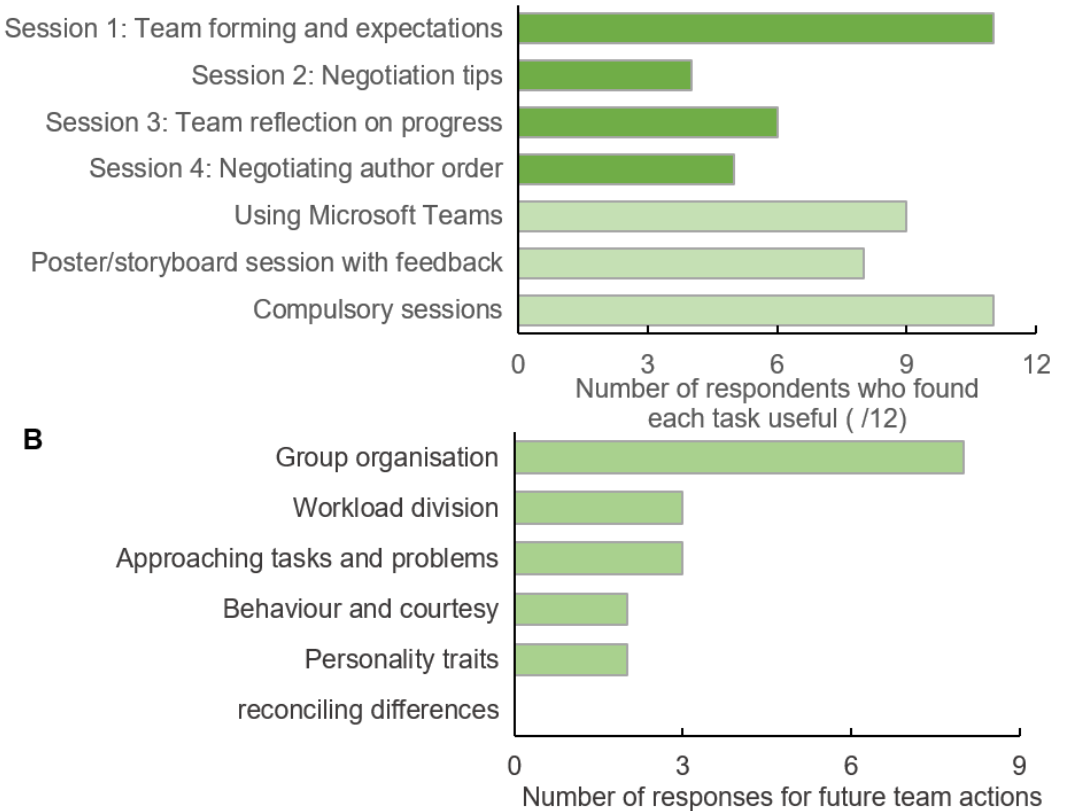

C

= not a lot $\square$ a little $\square$ a lot $=$ all the time
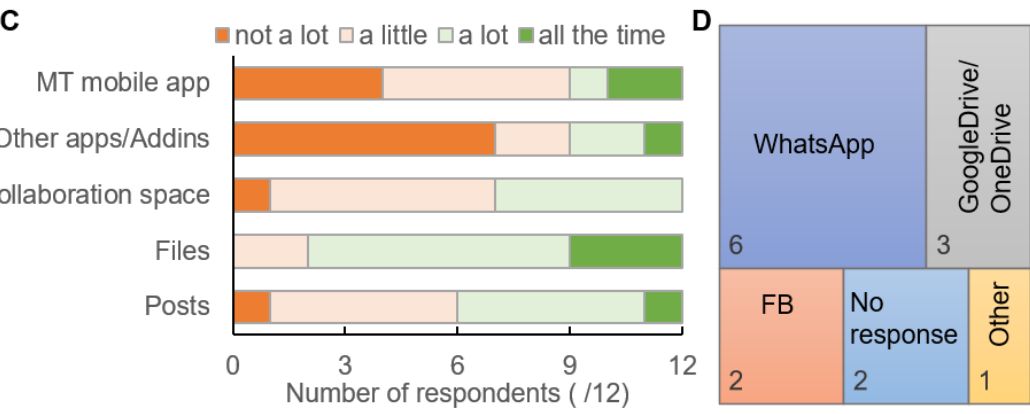

Figure 6: Student perspectives of structured team training program and use of collaborative technologies. (A) Number of respondents finding different aspects of the course useful: first four activities were explicit team training workshops, 'Using Microsoft Teams' was a tutorial on how to use the online collaboration software, the poster session was assessed and student were provided feedback on science content and video plan and the final point was the number of respondents who felt having 'Compulsory Sessions' was useful. B) Coded open-ended responses for what students would do differently in their next team experience. C) the amount that students used different Features within Microsoft Teams: 'MT (Microsoft Teams) mobile' application, 'Collaboration space' was within the built-in OneNote Class Notebook addin, 'Files' is the page where students can upload and share files and 'Posts' is where students can have text chat conversations (or video calls) with the other member of their Microsoft Team. (D) visual representation of the other collaboration technologies mentioned by students in an open-ended question - The size of each rectangle represents the proportion of responses allocated to that feature, the number of respondents is given in the lower left of each box, FB = Facebook Messenger. Total respondents $=12$; for $B$ and $D$ the total does not equal 12 because students may have mentioned more than one point in their response. 
A

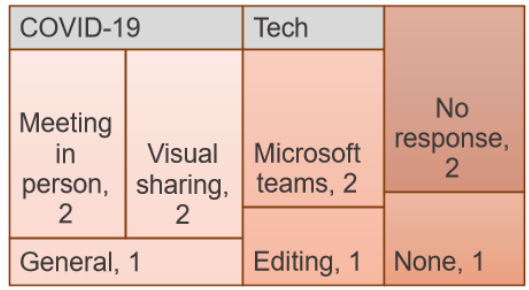

C

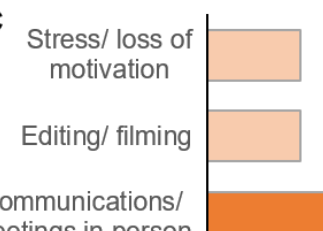

meetings in person
B

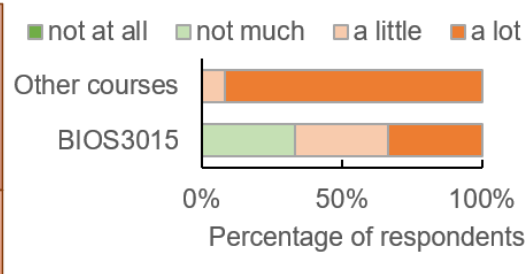

D

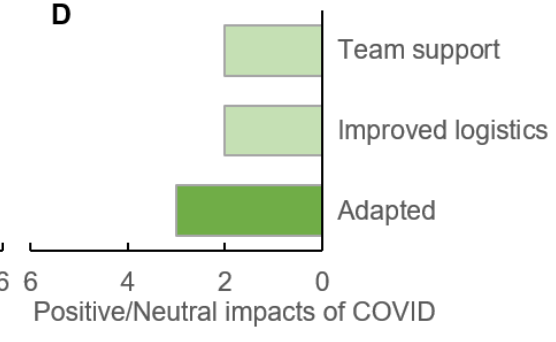

Figure 7: Student perspectives of COVID-19 challenges. When asked about any difficulties they faced completing the assignment, most comments were related to COVID-19 (A). The size of the boxes (A) represent the proportion of responses in that code category, the number of responses is given after each code name. B) Respondents perception of the impact of COVID-19 on Plants and the Soil Environment (BIOS3015) or their other courses. (C) the number of comments considered negative impacts of COVID-19 on their assignment for BIOS3015 and (D) the number of comments considered neutral or positive impacts of COVID-19 on their assignment. Total respondents $=12$; for A,C,D the total does not equal 12 because students may have mentioned more than one point in their response. 